\title{
Disks, accretion and outflows of brown dwarfs
}

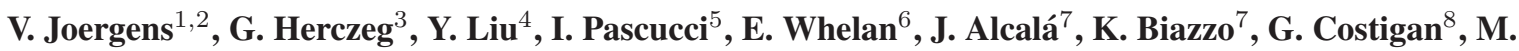

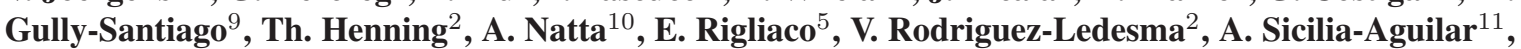 \\ J. Tottle ${ }^{12}$, and S. Wolf ${ }^{4}$ \\ ${ }^{1}$ Zentrum für Astronomie Heidelberg, Inst. für Theor. Astrophysik, Albert-Ueberle-Str. 2, 69120 Heidelberg, Germany \\ 2 Max-Planck Institut für Astronomie, Königstuhl 17, 69117 Heidelberg, Germany, viki@mpia.de \\ 3 Kavli Institute for Astronomy and Astrophysics, Peking University, Beijing, 100871, PR China \\ 4 University of Kiel, Institute of Theoretical Physics and Astrophysics, Leibnizstrasse 15, 24098 Kiel, Germany \\ ${ }^{5}$ Lunar \& Planetary Lab., Depart. of Planetary Sciences, Univ. of Arizona, 1629 E. University Blvd., Tucson, AZ 85721 \\ ${ }^{6}$ Dublin Institute for Advanced Studies, 10 Burlington Road, Dublin 4, Ireland \\ 7 INAF - Osservatorio Astronomico di Capodimonte, via Moiariello 16, 80131, Napoli, Italy \\ 8 European Southern Observatory, Karl-Schwarzschild-Str. 2, 85748 Garching, Germany \\ 9 Department of Astronomy, University of Texas at Austin, 1 University Station C1400, Austin, TX, 78712, USA \\ 10 Osservatorio Astrofisico di Arcetri, INAF, Largo E. Fermi 5, 50125 Firenze, Italy \\ 11 Departamento de Física Teórica, Facultad de Ciencias, Univ. Autónoma de Madrid, Cantoblanco, 28049 Madrid, Spain \\ 12 Imperial College London, 1010 Blackett Lab., Prince Consort Road, London SW7 2AZ, UK
}

\author{
Received XXXX, accepted XXXX
}

Published online XXXX

Key words brown dwarfs, stars: pre-main sequence, circumstellar matter, ISM: jets and outflows.

Characterization of the properties of young brown dwarfs are important to constraining the formation of objects at the extreme low-mass end of the initial mass function. While young brown dwarfs share many properties with solar-mass $\mathrm{T}$ Tauri stars, differences may be used as tests of how the physics of accretion/outflow and disk chemistry/dissipation depend on the mass of the central object. This article summarizes the presentations and discussions during the splinter session on Disks, accretion and outflows of brown dwarfs held at the CoolStars17 conference in Barcelona in June 2012. Recent results in the field of brown dwarf disks and outflows include the determination of brown dwarf disk masses and geometries based on Herschel far-IR photometry (70-160 $\mu \mathrm{m})$, accretion properties based on X-Shooter spectra, and new outflow detections in the very low-mass regime.

(C) 2013 WILEY-VCH Verlag GmbH \& Co. KGaA, Weinheim

\section{Introduction}

The exploration of disks, accretion and outflows of young brown dwarfs (BD) plays an important role in developing our understanding of BD formation, planet formation, and the physics of circumstellar disks and outflows in general. It is related to fundamental open questions in stellar astronomy, such as: Do BDs form via the same path as stars? Can planets form around BDs? How do disks develop in a lowgravity, -temperature, and -radiation environment?.

It has been established in the last years that BDs and very low-mass stars (VLMS) at an age of a few Myrs resemble higher mass $\mathrm{T}$ Tauri stars. They exhibit surface activity, such as cool spots (e.g., Joergens et al. 2003; RodríguezLedesma et al. 2009) and coronal activity (e.g., Stelzer et al. 2006). BD/VLMS have disks with a similar fraction as stars detected at mid-IR (e.g., Comerón et al. 2000; Natta \& Testi 2001; Jayawardhana et al. 2003; Luhman et al. 2008) and far-IR/submm wavelengths (e.g., Klein et al. 2003; Scholz et al. 2006; Harvey et al. 2012), which are actively accreting (e.g., White \& Basri 2003; Mohanty et al. 2005; Herczeg \& Hillenbrand 2008; Rigliaco et al. 2011), and often show signs of grain growth and crystallization (e.g., Apai et al. 2005; Pascucci et al. 2009). The finding that very young BDs rotate on average slower (e.g., Joergens \& Guenther 2001; Joergens et al. 2003; Caballero et al. 2004) than their older counterparts (e.g., Bailer-Jones \& Mundt 2001; Mohanty \& Basri 2003) is indicative of a magnetic braking mechanism due to interaction with the disk. Outflows from $\mathrm{BD} / \mathrm{VLMS}$ have been observed in spectro-astrometry of forbidden emission lines (e.g., Whelan et al. 2005, 2009; Bacciotti et al. 2011; Joergens et al. 2012a), and in images of CO J=2-1 emission (Phan-Bao et al. 2008).

Many details of the properties of BD disks and outflows, however, are still unclear: What are the masses and sizes of BD/VLMS disks? How does grain evolution occur in these disks? Do planets form in circum-BD disks? What are the properties of BD/VLMS outflows and how does the outflow mechanism work at low mass-accretion rates? Are there any discontinuities of all these properties with mass indicating a different formation path of BDs compared to stars? Several new instruments that are ideally suited to study disks and outflows of faint objects 
(PACS/Herschel, X-Shooter/VLT, ALMA-early science) are producing currently their first results. At the splinter session on Disks, accretion and outflows of brown dwarfs at the CoolStars17 conference (Barcelona, June 2012, www.mpia.de/homes/joergens/cs17splinter.html) leading scientists as well as young researchers presented and discussed recent results in the field of BD/VLMS disks and outflows, as outlined in the following.

\section{Accretion in BD disks}

Stars and BDs are thought to gain most of their mass through disk accretion. This process also regulates the angular momentum that the object receives from the surrounding interstellar medium. In addition, accretion measurements provide a crude probe of the gas content in the disk. In the current picture of magnetospheric accretion, material accreted through the disk is falling along magnetic accretion columns onto the stellar surface causing an accretion shock (e.g., Ghosh \& Lamb 1979; Königl 1991; Shu et al. 1993). For solar mass stars, the evidence for magnetospheric accretion in funnel flows is based primarily on long-term monitoring of line profiles of individual stars (Bouvier et al. 2007; Alencar et al. 2012), models of these line profiles (Kurosawa et al. 2008; Fischer et al. 2008), and models of how accretion would flow in magnetospheric structures constructed from Zeeman Doppler imaging (e.g., Gregory \& Donati 2011). The magnetospheric accretion geometry is assumed to be similar for BDs as for classical T Tauri stars (CTTS), with some support from models of $\mathrm{H} \alpha$ line profiles (Muzerolle et al. 2003, 2005).

Accretion rates, derived from the accretion luminosity,

$$
\dot{M}=\left(1-\frac{R_{*}}{R_{i n}}\right)^{-1} \frac{L_{a c c} R_{*}}{G M_{*}} \sim 1.25 \frac{L_{a c c} R_{*}}{G M_{*}}
$$

can be measured directly from the excess continuum emission, which is predominantly emitted from the shock regions, or indirectly from a variety of tracers, e.g. hydrogen and helium recombination lines, which are produced predominantly in the accretion funnel flows (Gullbring et al. 1998). VLT/X-Shooter observations covering the 300$2500 \mathrm{~nm}$ wavelength range demonstrate the reliability in using many different indirect measures of accretion luminosity, including $\mathrm{H} \mathrm{I}, \mathrm{He} \mathrm{I,} \mathrm{Na} \mathrm{I,} \mathrm{and} \mathrm{Ca} \mathrm{II} \mathrm{lines,} \mathrm{and} \mathrm{with} \mathrm{a}$ similar ratio of line to continuum luminosity across the mass range from BDs to stars (Rigliaco et al. 2012; Fig. 1).

Mass accretion rates for BDs are of the order of $10^{-9}$ to $10^{-12} M_{\odot} \mathrm{yr}^{-1}$ (e.g., Muzerolle et al. 2003; Natta et al. 2004; Mohanty et al. 2005; Herczeg et al. 2009). The accretion rate scales with the mass of the central object from stars of several solar masses to $0.1 M_{\odot}$ as $\dot{M} \propto M^{\alpha}$ with $\alpha=1.3$ 3 (Muzerolle et al. 2005; Natta et al. 2006; Garcia-Lopez et al. 2006; Fang et al. 2009; Biazzo et al. 2012, Manara et al. 2012). Recent multi-epoch spectroscopy of BD/VLMS in Cha I revealed a population of Class II sources with no significant accretion (Mohanty \& Tottle 2013, in prep.).

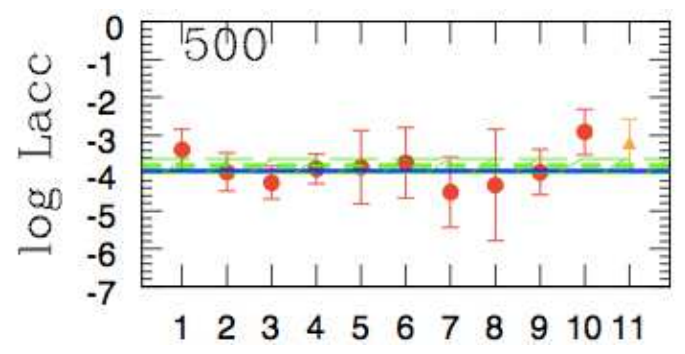

Fig. 1 Accretion indicators based on X-Shooter observations from Rigliaco et al. (2012). Displayed are the accretion luminosity Lacc derived for a BD based on 11 different accretion indicators $(1=\mathrm{H} \alpha, 2-\mathrm{H} \beta, 3=\mathrm{H} \gamma, 4=\mathrm{H} 9,5=\mathrm{Pa} \gamma, 6=\mathrm{Pa} \beta, 7=\mathrm{HeI}, 8=\mathrm{NaI}$, $9=\mathrm{Ca} 854,10=866,11=\mathrm{Uband})$; green dashed line: average Lacc computed considering the accretion tracers, blue solid line: Lacc from the excess continuum emission.

They can be separated into 3 classes: (1) edge-on disks, which artificially suppresses the $\mathrm{H} \alpha$ emission by disk occlusion of the accretion funnel flows/hot spots; (2) disks with large inner holes, in which accretion is truly quenched; and (3) flat anemic disks, in which the reduction of both the disk flux and accretion rate is presumably simply a result of evolution.

Variability is an essential part of the accretion process for T Tauri objects from solar to substellar masses (e.g., Stelzer et al. 2007). Study of its origin is important to understand the underlying physics and correctly interpret the observed signatures of e.g. accretion. Recent spectroscopic monitoring with FLAMES/VLT of 25 stars (G2-M5.75) in Cha I covering time-scales of weeks and months revealed variable accretion rates with average amplitudes of 0.89 dex based on $\mathrm{Ca}$ II and 0.41 dex based on $\mathrm{H} \alpha \mathrm{EW}$, resp. (Costigan et al. 2012). A significant portion of this accretion variability occurs on short timescales (8-25 days), which are of the order of the rotation rates of such objects (e.g., Joergens et al. 2003; Rodriguez-Ledesma et al. 2009), indicating that it probes the spatial structure in the accretion flows. In rare cases, large amplitude photometric variability in BDs is detected and attributed to periodic obscuration of the central object by the circum-BD or circumbinary disk (Looper et al. 2010; Cody \& Hillenbrand 2011; RodriguezLedesma et al. 2012). For the BD candidate CHS7797 (M6), this is shown impressively based on I-band monitoring covering 6 yrs as well as simultaneous monitoring in the I, J, Ks, IRAC [3.6], and [4.5] bands over 40 days (RodriguezLedesma et al. 2012).

\section{Disk masses and geometries of BDs}

The existence of dust disks around sub-stellar objects is a well-established phenomenon, but is mainly based on the detection of mid-IR excess emission (cf. Sect.1), which can probe only about the inner $\sim 0.1 \mathrm{AU}$ around BDs. The high sensitivity of the Herschel Space Observatory and its coverage of the far-IR portion of the wavelength regime (70, $160 \mu \mathrm{m}$ ) allows for the first time to trace cold dust (radial 


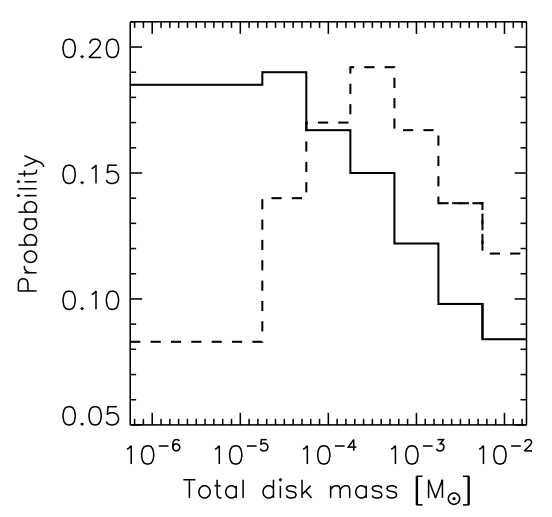

Fig. 2 Distribution of BD/VLMS disk masses determined based on SED fitting including Herschel data (from Harvey et al. 2012).

extents of $\sim 1 \mathrm{AU}$ ) and constrain the disk properties for a statistically significant sample of young BDs. A Herschel survey of $47 \mathrm{BD} / \mathrm{VLMS}$ (M3-M9.5) in young star-forming regions, for which there is evidence of circumstellar dust from Spitzer $(8-24 \mu \mathrm{m})$, detected $77 \%$ of them at $70 \mu \mathrm{m}$ and $30 \%$ at $160 \mu \mathrm{m}$ (Harvey et al. 2012). The disk properties that were derived based on modeling of their spectral energy distribution using radiative transfer codes (Wolf 2003; Pinte et al. 2009) showed that these BD/VLMS have very low disk masses $\left(10^{-6}-10^{-3} M_{\odot}=0.3 \mathrm{M}_{\oplus}-1 \mathrm{M}_{\text {Jup }}\right.$, Fig. 2) compared to disks of CTTS $\left(10^{-3}-10^{-1} M_{\odot}\right.$, e.g. Williams \& Cieza 2011). Such low disk masses were also derived for other BD/VLMS based on far-IR/submm data (e.g., Joergens et al. 2012a). While lower disk masses are expected for objects of lower mass, the Herschel survey hints that the ratio $\mathrm{M}_{d i s k} / \mathrm{M}$ could be smaller for BD/VLMS by a factor of 3-10 compared to that of CTTS and that they cover a wider range. The geometries (scale heights, flaring angles) of BD/VLMS disks are found to be consistent with those seen around CTTS, although they are not as flared as expected based on gas being in hydrostatic equilibrium (Szúcs et al. 2010).

The disk masses from Harvey et al. (2012) may be affected by a potential bias towards weak BD disks in the Herschel sample because of selection against targets in regions covered by Herschel's large scale shallow surveys. The dust masses also rely on the standard assumption of a gas-todust ratio of 100. While Harvey et al. (2012) show that at $\geq 160 \mu \mathrm{m}$, the disks are typically optically thin over most of the radii emitting at those wavelengths, ALMA observations will trace colder dust than observed with Herschel and may also provide direct measurements of the gas content in disks and will, therefore, be important to determine both the masses and sizes of BD disks.

\section{Evolution of gas and grains in BD disks}

There is a growing observational evidence that disks around stars of different masses evolve differently. Dust disk lifetimes, as measured from the presence of IR excess emis-

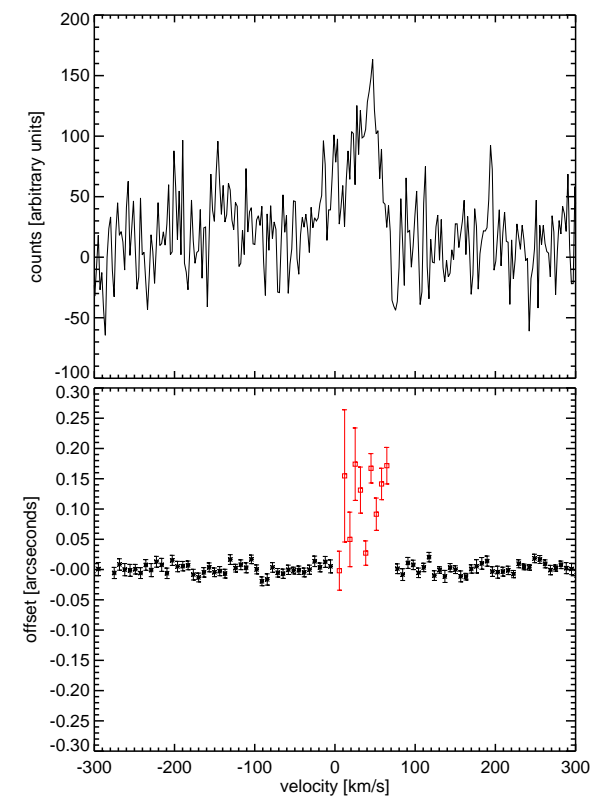

Fig. 3 Discovery of an asymmetric outflow of a VLMS star (M5) by spectro-astrometry of forbidden emission of [S II] $\lambda 6716$ (from Joergens et al. 2012b). Top: Line profile. Bottom: Spatial offset vs. radial velocity of the continuum (black asterisks) and of the continuum subtracted FEL (red squares) demonstrating that the [S II] line is emitted at a distance of up to 170 mas $(\sim 30 \mathrm{AU})$ from the central source with velocities of up to $65 \mathrm{~km} \mathrm{~s}^{-1}$.

sion, appear to be longer toward lower mass stars and BDs (Carpenter et al. 2006; Luhman 2009). These comparisons rely on identification of diskless BDs, including those found in ongoing surveys that target water absorption bands in BD atmospheres (Gully-Santiago et al. 2013, in prep). The processes of grain growth and dust settling, which are the first steps toward planet formation, appear, on the contrary, to occur faster in disks around BD/VLMS (Apai et al. 2005; Allers et al. 2006; Pascucci et al. 2009; Riaz 2009; Szúcs et al. 2010; Riaz et al. 2012). This is based on the finding of differences in the scale-height of the gas (from the temperature distribution) and that of the grains (from the SED) between CTTS and BD. While the evolution of the gas is not constrained, the steep dependence between mass accretion rate and mass of the central star (cf. Sect. 2) could be the sign of a faster disk clearing around BD/VLMS. In the picture proposed by Hartmann et al. (2006) these disks are fully magnetically active (unlike disks around sun-like stars that have dead zones), hence they accrete faster. Finally, we discussed the chemistry occurring on the surface of BD disks, with emphasis on the low abundance of $\mathrm{HCN}$ and the depletion of water with respect to disks around sun-like stars (Pascucci et al. 2009, Pascucci et al. 2013, in prep).

\section{Outflows of BDs}

The launch and driving of jets and outflows is a key process in the formation phase. Outflows carry away both mass and 
angular momentum from the star-disk system (e.g., Launhardt et al. 2009). In recent years, outflows driven by objects as low-mass as BD/VLMS were found; the majority of these are optical outflows discovered by spectro-astrometry of forbidden emission lines (FELs) formed in shocks (e.g., Whelan et al. 2005, 2009; Bacciotti et al. 2011; Joergens et al. 2012a), alongside a spatially resolved molecular outflow detected in the CO J=2-1 transition (Phan-Bao et al. 2008, 2011). While this research is in an early stage and the number of BD/VLMS outflows confirmed by spatial information (spectro-astrometry or direct resolution) is still statistically small $(<10)$, comparison between CTTS and $\mathrm{BD} / \mathrm{VLMS}$ outflows has yielded a number of interesting results (Whelan et al. 2009; Bacciotti et al. 2011; Joergens et al. 2012a). Similarities between outflows of CTTS and BDs include, the observation of asymmetries (Joergens et al. 2012b; Fig. 3), both low- and high-velocity emission (Whelan et al. 2009), and jet collimation (Whelan et al. 2012). Initial measurements of the ratio between the mass outflow and accretion rates $\dot{M}_{\text {out }} / \dot{M}_{a c c}$ in BD/VLMS (Comerón et al. 2003; Whelan et al. 2009; Bacciotti et al. 2011) hint that it might be higher than the 5-10\% in CTTS (e.g., Hartigan et al. 1995; Sicilia-Aguilar et al. 2010; Fang et al. 2009). If this is confirmed, it would point to different efficiencies in the mechanisms at work to launch outflows at very low masses or very low mass accretion rates. The molecular outflow detected by Phan-Bao et al. (2008) is around an optically visible VLMS, i.e. an assumed counterpart to a Class II CTTS, while molecular outflows in the stellar regime are usually associated with Class 0 and Class I objects. The colder environment of BD/VLMS may mean that more molecular material is available in the later stages of evolution and, therefore, that $\mathrm{CO}$ outflows will be more commonly observed. The work on BD/VLMS outflows is now at a stage where techniques for detecting them are well developed and understood. First results have brought up some interesting questions, which need to be addressed through detections and study of BD/VLMS outflows for a larger number of objects.

Acknowledgements. VJ acknowledges funding by the ESF in Ba.Wü. YL acknowledges funding by the German Academic Exchange Service and the Research Unit FOR 795.

\section{References}

Alencar, S.H.P., Bouvier, J., Walter, F.M. et al. 2012, A\&A, 541, 116

Allers, K. N., Kessler-Silacci, J. E., Cieza, L. A., Jaffe, D. T. 2006, ApJ, 644, 364

Apai, D., Pascucci, I., Bouwman, J., Natta, A., Henning, Th., Dullemond, C. P. 2005, Science, 310, 834

Bacciotti, F., Whelan, E.T., Alcalá, J.M. et al. 2011, ApJ, 737, L26

Bailer-Jones, C.A.L., Mundt, R. 2001, A\&A, 367, 218

Biazzo, K., Alcalá, J.A., Covino, E., Frasca, A., Getman, F., Spezzi, L., 2012, A\&A, 547, A104

Bouvier, J., Alencar, S.H.P., Boutelier, T. et al. 2007, A\&A, 463, 1017

Caballero, J.A., Béjar, V.J.S., Rebolo, R., Zapatero Osorio, M.R. 2004, A\&A, 424, 857
Carpenter, J. M., Mamajek, E. E., Hillenbrand, L. A., Meyer, M. R. 2006, ApJ, 651, L49

Cody, A.M., \& Hillenbrand, L.A. 2011, ApJ, 191, 389

Comerón, F., Neuhäuser, R., Kaas, A.A. 2000, A\&A, 359, 269

Comerón, F., Fernández, M., Baraffe, I., Neuhäuser, R., Kaas, A. A. 2003, A\&A, 406, 1001

Costigan, G., Scholz, A., Stelzer, B., Ray, T., Vink, Mohanty, S. 2012, MNRAS, in press

Fang, M., van Boekel, R., Wang, W., Carmona, A., Sicilia-Aguilar, A., Henning, Th. 2009, A\&A, 504, 461

Fischer, W., Kwan, J., Edwards, S., Hillenbrand, L. 2008, ApJ, 687,1117

Garcia-Lopez, R., Natta, A., Testi, L., Habart, E. 2006, A\&A, 459, 837

Ghosh, P., Lamb, F.K. 1979, ApJ, 234, 296

Gregory, S.G., Donati, J.-F. 2011, AN, 332, 1027

Gullbring, E., Hartmann, L., Briceno, C., Calvet, N. 1998, ApJ, 492, 323

Hartigan, P, Edwards, S., \& Ghandour, L. 1995, ApJ, 452, 736

Hartmann, L., D’Alessio, P., Calvet, N., Muzerolle, J. 2006, ApJ, 648, 484

Harvey, P.M., Henning, Th., Liu, Y. et al. 2012, ApJ, 755, 67

Herczeg, G. J., Hillenbrand, L. A. 2008, ApJ, 681, 594

Herczeg, G.J., Cruz, K.L., Hillenbrand, L.A. 2009, ApJ, 696, 1589

Jayawardhana, R., Ardila, D.R., Stelzer, B., Haisch Jr., K.E. 2003, AJ, 126, 1515

Joergens, V., Guenther, E. 2001, A\&A, 379, L9

Joergens, V., Fernández, M., Carpenter, J. M., Neuhäuser, R. 2003, ApJ, 594, 971

Joergens, V., Pohl, A., Sicilar-Aguilar, A., Henning, Th. 2012a, A\&A, 543, A151

Joergens, V., Kopytova, T., Pohl, A. 2012b, A\&A, 548, A124

Klein, R., Apai, D., Pascucci, I., Henning, Th., Waters, L. B. F. M. 2003, ApJ, 593, L57

Königl, A. 1991, ApJ, 370, L39

Kurosawa, R., Romanova, M.M., Harries, T.J. 2008, MNRAS, 385,1931

Launhardt, R., Pavlyuchenkov, Ya, Gueth, F. et al. 2009, A\&A, 494, 147

Looper, D.L., et al. 2010, ApJ, 714, L45

Luhman, K.L., Allen, L.E., Allen, P.R. et al. 2008, ApJ, 675, 1375

Luhman, K. L. 2009, In: Cool stars, stellar systems and the Sun, AIP Conf. Proc., 1094, 55

Manara, C.F., Robberto, M., Da Rio, N., et al. 2012, ApJ, 755, 154

Mohanty, S., Basri, G. 2003, ApJ, 583, 451

Mohanty, S., Jayawardhana, R., Basri, G. 2005, ApJ, 626, 498

Muzerolle, J., Hillenbrand, L., Calvet, N., Briceño, C., Hartmann, L. 2003, ApJ, 592, 266

Muzerolle, J., Luhman, K.L., Briceno, C., Hartmann, L., Calvet, N. 2005, ApJ, 625, 906

Natta, A., Testi, L. 2001, A\&A, 376, L22

Natta, A., Testi, L., Muzerolle, J., Randich, S., Comerón, F., Persi, P. 2004, A\&A, 424, 603

Natta, A., Testi, L., Randich, S. 2006, A\&A, 452, 245

Pascucci, I., Apai, A., Luhman, K. et al. 2009, ApJ, 696, 143

Phan-Bao, N., Riaz, B., Lee, C.-F. et al. 2008, ApJ, 689, L141

Phan-Bao, N., Lee, C.-F., Ho, P.T.P., Tang, Y.-W. 2011, ApJ, 735, 14

Pinte, C. et al. 2009, A\&A, 498, 967

Riaz, B. 2009, ApJ, 701, 571

Riaz, B., Honda, M., Campins, H. et al. 2012, MNRAS, 420, 2603

Rigliaco, E., Natta, A., Randich, S. et al. 2011, A\&A, 526, L6

Rigliaco, E., Natta, A., Testi, L. et al. 2012, A\&A, in press 
Rodríguez-Ledesma, M. V., Mundt, R., Eislöffel J. 2009, A\&A, 502,883

Rodríguez-Ledesma, M. V., Mundt, R., Ibrahimov, M. et al. 2012, A\&A, 544, A112

Scholz, A., Jayawardhana, R., Wood, K. 2006, ApJ, 645, 1498

Shu, F., Najita, J., Galli, D., Ostriker, E., Lizano, S. 1993, In: Protostars and planets III, (A93-42937 17-90), p. 3

Sicilia-Aguilar, A., Henning, Th., Hartmann, L. W. 2010, ApJ, 710,597

Stelzer, B., Micela, G., Flaccomio, E., Neuhäuser, R., Jayawardhana, R. 2006, A\&A, 448, 293

Stelzer, B., Scholz, A., Jayawardhana, R. 2007, ApJ, 671, 842

Szúcs, L., Apai, D., Pascucci, I., Dullemond, C. P. 2010, ApJ, 720, 1668

Whelan, E.T., Ray, T.P., Bacciotti, F., Natta, A., Testi, L., Randich, S. 2005, Nature, 435, 652

Whelan, E.T., Ray, T.P., Podio, L., Bacciotti, F., Randich, S. 2009, ApJ, 706, 1054

White, R.J., \& Basri, G. 2003, ApJ, 582, 1109

Whelan, E. T. , Ray, T., Comerón, F., Bacciotti, F., Kavanagh, P. 2012, ApJ, in press

Williams, J.P., Cieza, L.A. 2011, ARA\&A, 49, 67

Wolf, S. 2003, ApJ 582, 859 